\title{
Novel oxygen saturation imaging endoscopy to assess anastomotic integrity in a porcine ischemia model
}

\author{
Hiro Hasegawa ${ }^{1,2}$, Nobuyoshi Takeshita ${ }^{1,2}$ and Masaaki Ito ${ }^{1,2^{*}}$ (1)
}

\begin{abstract}
Background: Establishing anastomotic integrity is crucial for avoiding anastomotic complications in colorectal surgery. This study aimed to evaluate the safety and feasibility of assessing anastomotic integrity using novel oxygen saturation imaging endoscopy in a porcine ischemia model.

Methods: In three pigs, a new endoscope system was used to check the mechanical completeness of the anastomosis and capture the tissue oxygen saturation $\left(\mathrm{StO}_{2}\right)$ images. This technology can derive the $\mathrm{StO}_{2}$ images from the differences in the absorption coefficient in the visible light region between oxy- and deoxy-hemoglobin. Bowel perfusion at the proximal rectum was assessed before and after the anastomosis, and $1 \mathrm{~min}$ and $30 \mathrm{~min}$ after the ligation of the cranial rectal artery (CRA).

Results: The completeness of the anastomoses was confirmed by the absence of air leakage. Intraluminal oxygen saturation imaging was successfully performed in all animals. There was no significant difference in the $\mathrm{StO}_{2}$ level before and after the anastomosis ( $52.6 \pm 2.0$ vs. $52.0 \pm 2.6 ; p=0.76$, respectively). The $\mathrm{StO}_{2}$ level of the intestine on the oral side of the anastomosis one minute after the CRA ligation was significantly lower than immediately after the anastomosis ( $15.9 \pm 6.0$ vs. $52.0 \pm 2.6 ; p=0.006$, respectively). There was no significant difference in the $\mathrm{StO}_{2}$ level between 1 min after and 30 min after the CRA ligation ( $15.9 \pm 6.0$ vs. $12.1 \pm 5.3 ; p=0.41$, respectively).
\end{abstract}

Conclusion: Novel oxygen saturation imaging endoscopy was safe and feasible to assess the anastomotic integrity in the experimental model.

Keywords: Anastomotic integrity, Endoscopy, Perfusion assessment, Tissue oxygen saturation

\section{Background}

Despite advances in surgical techniques and medical devices, anastomotic leakage and stricture remain one of the most dreaded complications in colorectal surgery. Anastomotic complications, such as postoperative morbidity and mortality, have a negative impact on short-term outcomes $[1,2]$. In addition, they also affect

\footnotetext{
*Correspondence: maito@east.ncc.go.jp

${ }^{1}$ Department of Colorectal Surgery, National Cancer Center Hospital East,

6-5-1 Kashiwanoha, Kashiwa, Chiba 277-8577, Japan

Full list of author information is available at the end of the article
}

long-term outcomes, such as survival in cancer patients [3] and quality of life [4].

Various risk factors have been reported to be associated with anastomotic leakage in colorectal surgery [1, 5]. Male sex, low anastomotic level (within $5 \mathrm{~cm}$ from the anal verge), and multiple firings with a linear stapler are the important factors for anastomotic leakage. Macroscopic intraluminal assessment of anastomotic completeness using an intraoperative colonoscopy and the air leakage test have been broadly used to check the incompleteness of the anastomosis during surgery [6-9]. It is also a vital procedure for avoiding anastomotic leakage to 
evaluate whether blood supply to the anastomotic site is sufficient $[5,10]$. Recent technological advancement has successfully enabled real-time visualization of blood perfusion at the anastomotic site using indocyanine green fluorescence angiography (ICG-FA) in colorectal surgery [10]. ICG-FA has been widely used to evaluate anastomotic perfusion and it has been reported that it could reduce the incidence of anastomotic leakage in rectal surgery [10]. Conversely, there are also negative aspects such as the necessity of administering the fluorescent substance, possibility of ICG allergy, and the surgeons' qualitative assessment.

If intraoperative endoscopy could enable the surgeon to assess blood perfusion of the anastomosis similar to that via a fluorescence scope, it would be a useful method for assessing not only blood perfusion, but also for obtaining a macroscopic view of the anastomotic line and the realtime status of the air leak test during colorectal surgery.

A novel endoscope system was therefore developed to assess the bowel perfusion in real time by capturing the tissue oxygen saturation $\left(\mathrm{StO}_{2}\right)$ images $[11,12]$. This system provides a repeated, quantitative assessment of bowel perfusion without the need for administering the fluorescent dye. Additionally, the system enables direct visualization of the anastomosis and allows the surgeon to conduct an air leak test to confirm mechanical completeness. The aim of the current study was to evaluate the feasibility of assessing the anastomotic integrity using a novel oxygen saturation imaging endoscopy in a porcine ischemia model.

\section{Methods}

\section{Animals}

The animals were obtained commercially from KAC Company Limited (Kyoto, Japan). A total of three pigs (domestic, female, crossbred with Large Yorkshire and Landrace, mean weight $47.2 \pm 0.6 \mathrm{~kg}, 3$ to 4 months of age) were used in the current non-survival study. The study protocol was approved by the Committee for Ethics of Animal Experimentation of the National Cancer Center (Japan) (K18-022). Experiments were performed according to the Guidelines for Animal Experiments (Science Council of Japan: Guidelines for Proper Conduct of Animal Experiments, 2006) and Animal Research: Reporting of In Vivo Experiments (ARRIVE) guidelines [13].

\section{Novel oxygen saturation imaging endoscope system}

A novel endoscope system EP-0002 for gastroenterological flexible endoscopy (FUJIFILM Corporation, Kanagawa, Japan) was used to capture the $\mathrm{StO}_{2}$ images (Fig. 1) [11, 12]. The EP-0002 provides the conventional observation modes of white light imaging (WLI).

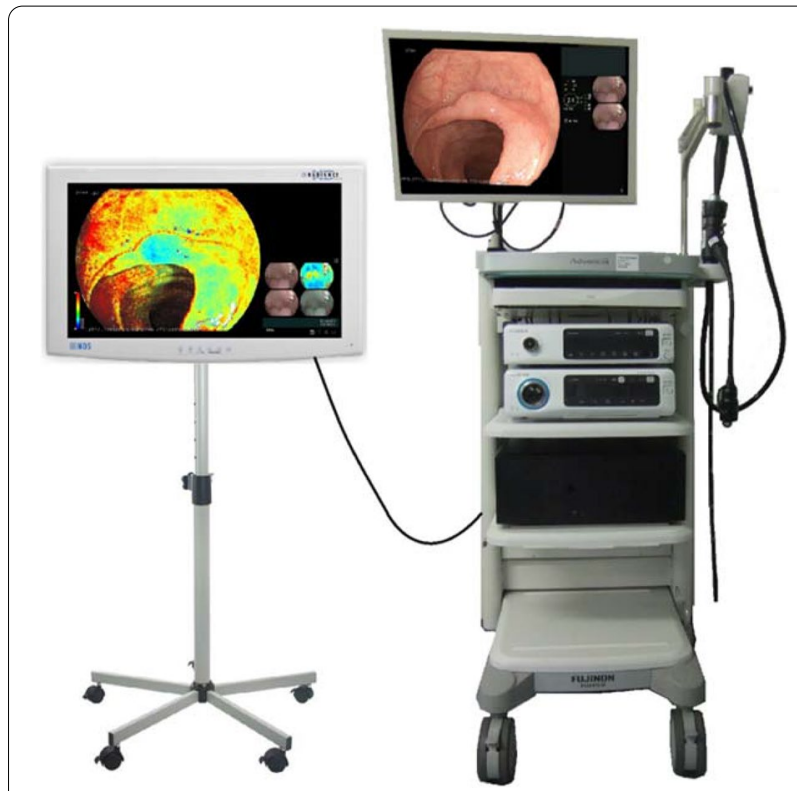

Fig. 1 Novel oxygen saturation imaging endoscope system (printed from FUJIFILM Corporation, with permission)

Additionally, the system provides a mode of oxygen saturation imaging (OSI), where the images of $\mathrm{StO}_{2}$ distribution in the tissue being observed can be obtained in real time. The WLI and OSI modes can be displayed simultaneously on separate monitors (Fig. 1). The OSI technology can derive the $\mathrm{StO}_{2}$ images from the differences in the absorption coefficient in the visible light region between oxy- and deoxy-hemoglobin using a small number of wavelengths (Fig. 2a). The light source is equipped with three types of laser diodes $(405,445$, and $473 \mathrm{~nm}$ ) for illumination. The laser light is guided to the tip of the endoscope, which excites the phosphors mounted at the tip to create white fluorescent light. The 405 and $445 \mathrm{~nm}$ laser light is used in the WLI mode. In the OSI mode, the unit alternately emits 445 and $473 \mathrm{~nm}$ light synchronously with the video frames at a speed of 30 frames per second (fps). Images are acquired with a red-green-blue (RGB) color charge-coupled device mounted at the tip of the endoscope. The dominant wavelength ranges of the sensitivity of the RGB image sensor are overlaid on both graphs (Fig. 2b). The processor unit controls the whole system and performs image processing for the conventional modes of WLI. In the OSI mode, the processor unit acquires two types of images sequentially and alternately that correspond to the illumination induced by the 445 and $473 \mathrm{~nm}$ lasers, respectively (Fig. 2c). The processor unit alternately extracts frames of $445 \mathrm{~nm}$ light from the input video signals and uses them to display the WLI images on the processor's monitor at a speed of $15 \mathrm{fps}$ (Fig. 2c). At the same time, the processor unit 

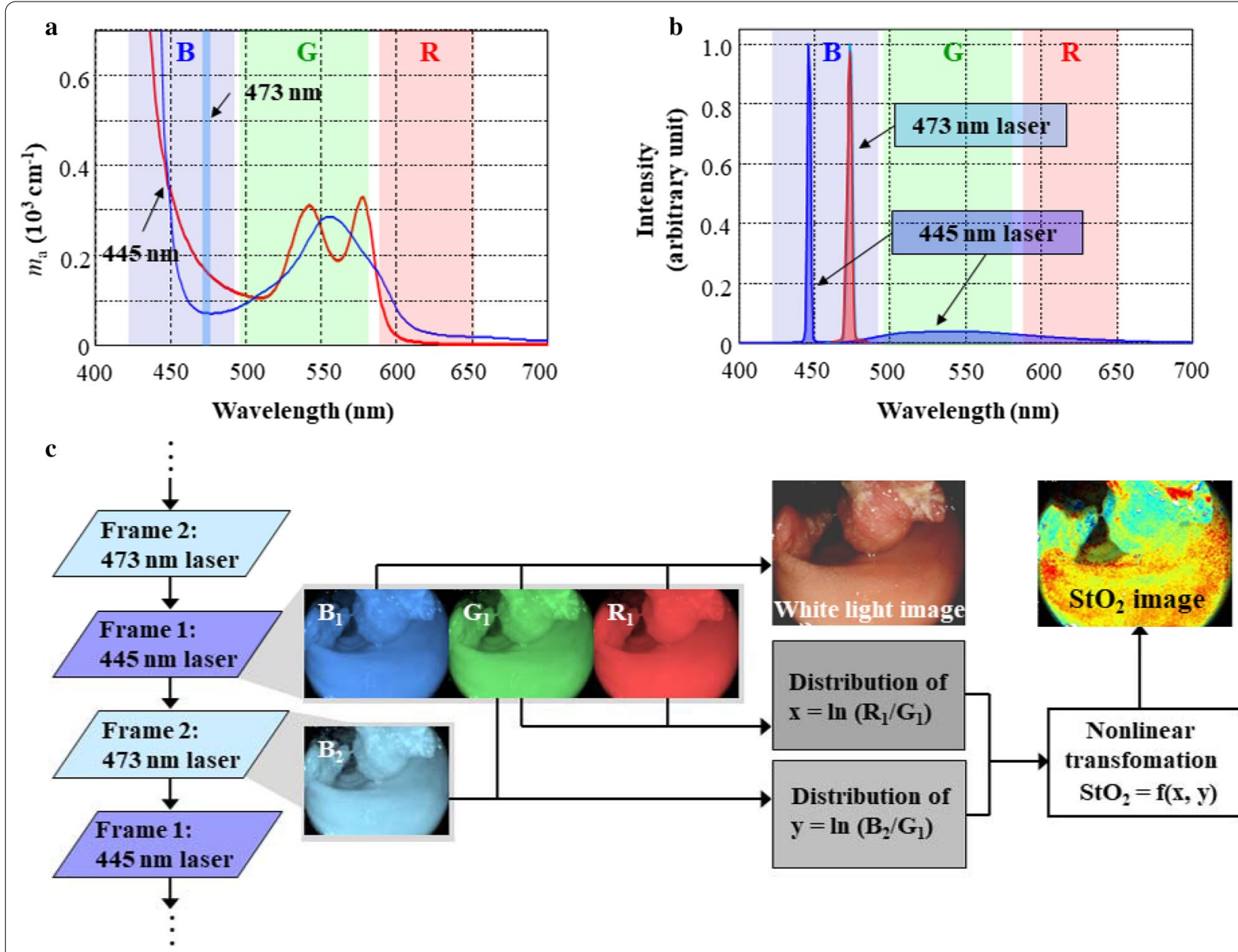

Fig. 2 Mechanism of tissue oxygen saturation imaging

transfers the video signals to the image processing (IP) unit, and the IP unit creates an $\mathrm{StO}_{2}$ image by using two consecutive frames corresponding to 445 and $473 \mathrm{~nm}$ illuminations (Fig. 2c). The $\mathrm{StO}_{2}$ images are displayed by assigning the $\mathrm{StO}_{2}$ level to the RGB display color of the monitor in $1 \%$ increments, with the $\mathrm{StO}_{2}$ level of $100 \%$ as red, $50 \%$ as yellowish green, and $0 \%$ as dark blue. The $\mathrm{StO}_{2}$ images are displayed on the IP's monitor at a speed of 15 fps almost in real time (Fig. 1).

\section{Study protocol}

After premedication with intramuscular injections of ketamine $(10 \mathrm{mg} / \mathrm{kg})$ and xylazine $(2 \mathrm{mg} / \mathrm{kg})$, general anesthesia was induced using 5\% isoflurane. The airway was secured via endotracheal intubation, and general anesthesia was maintained with 1 to $3 \%$ isoflurane. The pigs were mechanically ventilated with the following settings: tidal volume, 10 to $15 \mathrm{ml} / \mathrm{kg}$; respiratory rate, 12 breaths per minute; inspiratory to expiratory ratio, 1:2; positive end-expiratory pressure, $5 \mathrm{cmH}_{2} \mathrm{O}$; and fraction of inspired oxygen, 40\%. Ringer's lactate solution was administered intravenously through the auricular vein at 5 to $10 \mathrm{ml} / \mathrm{kg} / \mathrm{h}$. Heart rate, blood pressure, body temperature, and saturation of percutaneous oxygen $\left(\mathrm{SpO}_{2}\right)$ were monitored throughout the procedure. An electrically heated mat was placed below each pig to prevent hypothermia.

Under general anesthesia, a midline laparotomy was performed. The marginal vessels of the rectum were dissected at the level of the planned anastomotic site, and the rectum was transected using a linear stapler $(\mathrm{ECH}-$ ELON FLEX ENDOPATH Stapler with a gold cartridge; Ethicon Endo-Surgery Inc, Cincinnati, OH, USA). The anvil was placed in the proximal side of the rectum, and end-to-end anastomosis was performed with the double stapling technique using a $25 \mathrm{~mm}$ diameter circular stapler (PROXIMATE ILS Curved Intraluminal Stapler; Ethicon Endo-Surgery Inc, Cincinnati, OH, USA). After 
the anastomosis, an air leak test was performed to check the completeness of the anastomosis via the endoscope. The root of the cranial rectal artery (CRA: equivalent to the superior rectal artery in humans) was dissected, and the porcine ischemia model was created in three pigs (Fig. 3). Prior to the assessment, the intestinal lumen was washed with saline to remove the stool. The contralateral side of the mesentery of the proximal rectum was marked with suture in each of the pigs. Bowel perfusion at the proximal side of the rectum was assessed intraluminally using a novel OSI endoscopy before and immediately after the anastomosis, as well as $1 \mathrm{~min}$ and $30 \mathrm{~min}$ after CRA ligation.

At the end of the procedure, the pigs were sacrificed by a rapid intravenous injection of a lethal dose of potassium chloride (75 to $150 \mathrm{mg} / \mathrm{kg}$ ) while under deep general anesthesia using 5\% isoflurane, and asystole was confirmed by an electrocardiogram.

\section{Quantitative evaluation of oxygen saturation imaging}

Endoscopic procedures were recorded on videos and the stored still images were used to quantitatively evaluate the OSI with custom-made analysis software developed by FUJIFILM Corporation. A square region of interest was placed at three sites on the contralateral side of the mesentery of the proximal rectum in each of the pigs and each average value was calculated as the $\mathrm{StO}_{2}$ level. The $\mathrm{StO}_{2}$ levels were calculated at the following four time points: before and immediately after the anastomosis, and $1 \mathrm{~min}$ and $30 \mathrm{~min}$ after CRA ligation. The average $\mathrm{StO}_{2}$ levels of all three pigs were compared among the different time points.

\section{Statistical analysis}

Statistical analyses were performed using JMP version 15.1.0 (SAS Institute, Cary, NC, USA). Values are

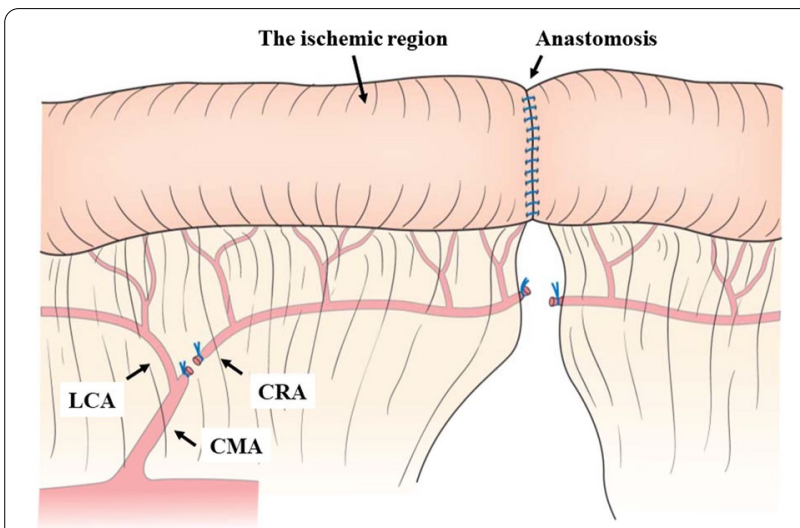

Fig. 3 A schematic diagram of the porcine ischemia model. CMA caudal mesenteric artery, CRA cranial rectal artery, LCA left colic artery presented as the mean \pm standard deviation. For comparing the $\mathrm{StO}_{2}$ levels, a paired $t$-test was utilized. $p$-values $<0.05$ were considered statistically significant.

\section{Results}

Heart rate was $100 \pm 20$ beats per minute, mean arterial pressure was $58 \pm 14 \mathrm{mmHg}$, body temperature was $36.6 \pm 0.6^{\circ} \mathrm{C}$, and $\mathrm{SpO}_{2}$ was $97 \pm 2.4$, which were stable throughout the experiments. All procedures including endoscopic and surgical steps were uneventful. Establishment of the bowel ischemia model was systematically reproduced in all pigs.

The status of the anastomosis was assessed intraluminally using the current endoscope after creating the anastomosis; there were no macroscopic findings of air leakage in any of the pigs. Oxygen saturation imaging was successfully performed in all animals. Before CRA ligation, the $\mathrm{StO}_{2}$ map of the proximal rectum showed a high $\mathrm{StO}_{2}$ value (Fig. 4). Immediately after CRA ligation, the $\mathrm{StO}_{2}$ map showed the appearance of a hypoxic area (Fig. 5). The hypoxic area was completely visible on the $\mathrm{StO}_{2}$ map and it corresponded to the ischemic regions. Bowel perfusion at the distal side of the rectum after dissection of marginal vessels showed a low $\mathrm{StO}_{2}$ value in all pigs.

Before the anastomosis, the mean $\mathrm{StO}_{2}$ level of the proximal rectum was $52.6 \pm 2.0$. Immediately after the anastomosis, the mean $\mathrm{StO}_{2}$ level was $52.0 \pm 2.6$. One minute after CRA ligation, the mean $\mathrm{StO}_{2}$ level decreased considerably to $15.9 \pm 6.0$; $30 \mathrm{~min}$ after CRA ligation, the mean $\mathrm{StO}_{2}$ level was 12.1 \pm 5.3 .

There was no significant difference in the $\mathrm{StO}_{2}$ level before and immediately after the anastomosis $(p=0.76$; Fig. 6). The $\mathrm{StO}_{2}$ level $1 \mathrm{~min}$ after CRA ligation was significantly lower than immediately after the anastomosis $(p=0.006$; Fig. 6). There was no significant difference in the $\mathrm{StO}_{2}$ level between 1 and 30 min after CRA ligation $(p=0.41$; Fig. 6).

\section{Discussion}

Anastomotic leakage and stricture remain one of the most dreaded complications in colorectal surgery. To the best of our knowledge, this is the first study to evaluate the safety and feasibility of assessing anastomotic integrity using novel OSI endoscopy in an experimental porcine model. All procedures were safely performed without any adverse events, and the anastomotic assessment was successful in all animals. Regarding the vital signs, the standard deviation of heart rate and mean arterial pressure was relatively large, but did not affect the results. Using this system, the macroscopic statuses regarding the completeness and hemostasis achieved 

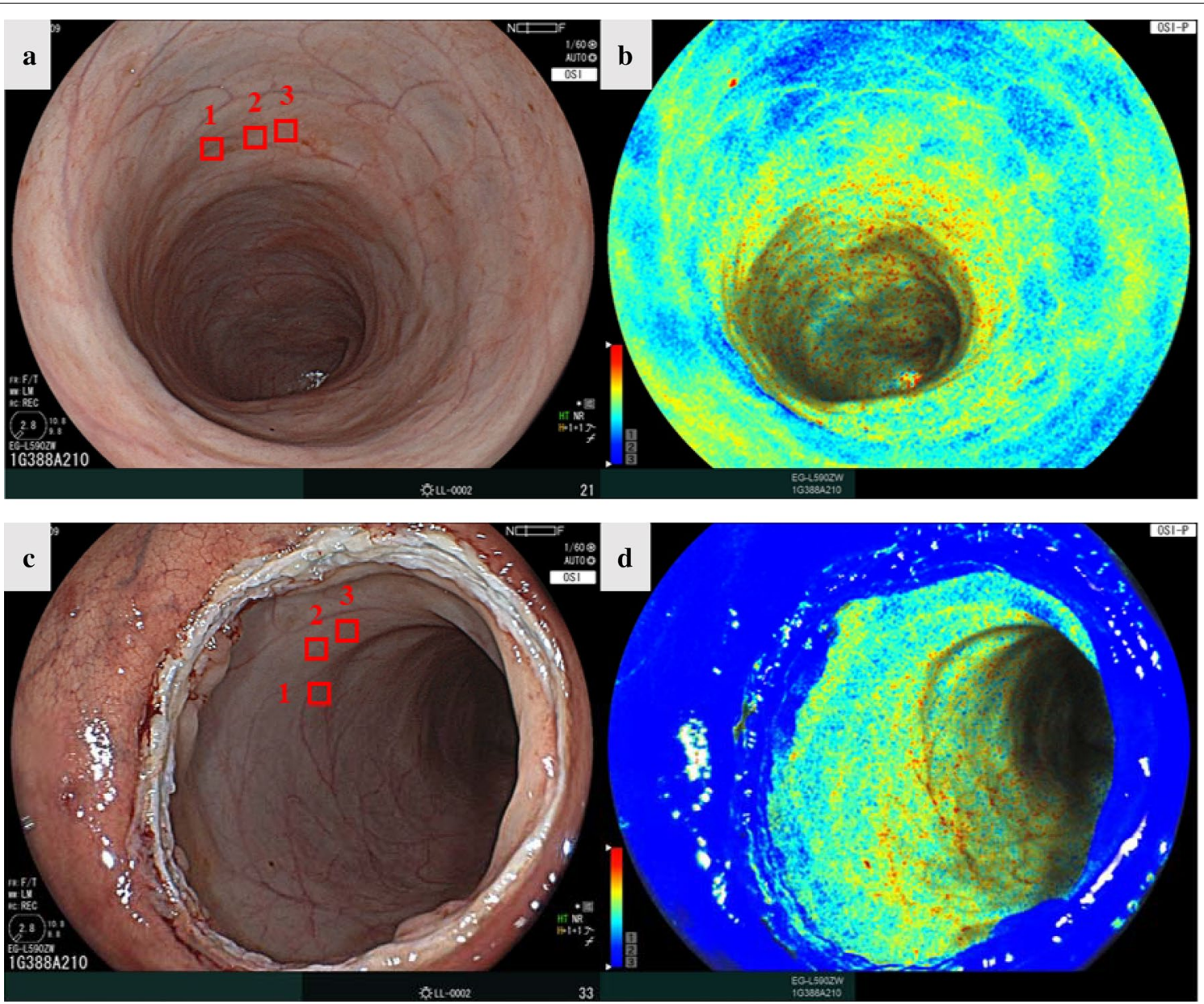

Fig. 4 a White light image before the anastomosis. $\mathbf{b} \mathrm{StO}_{2}$ map before the anastomosis. $\mathbf{c}$ White light image after the anastomosis. $\mathbf{d} \mathrm{StO}_{2}$ map after the anastomosis. $\mathrm{StO}_{2}$ tissue oxygen saturation

through the anastomosis could be directly checked not only by the intraluminal visualization, but also by an air leak test. Moreover, the bowel blood perfusion was repeatedly and quantitatively assessed without requiring the administration of fluorescent agents.

Anastomotic complications include patient-related, disease-related, and operative-related risk factors $[1,5]$. Among these factors, operative-related risk factors can be controlled by the surgeons; anastomotic integrity is considered to be the most important factor to reduce anastomotic complications [6]. In particular, sufficient blood supply to the anastomosis and complete mechanical attachment (e.g., with staples) are essential in order to avoid the abovementioned complications [5, 6]. However, to the best of our knowledge, there is no medical device that can evaluate these elements simultaneously and in real time.
Recent image navigation technology has improved some postoperative outcomes; it is expected to expand to more clinical fields in the future. ICG-FA is an excellent example of this; it could be a safe and useful method to assess bowel perfusion intraoperatively in real time and reduce the incidence of anastomotic leakage by selecting an appropriate intestine with sufficient blood perfusion during colorectal surgery [10]. However, this method has several disadvantages. First, ICG-FA cannot confirm the macroscopic completeness of the anastomosis from inside the intestine. Secondly, ICG-FA requires the administration of ICG as a fluorescent substance; ICG takes a long time to be fully excreted from the body, which makes it difficult to repeatedly assess bowel perfusion. Third, the issue of ICG allergy persists. Fourth, the assessment of ICG-FA is qualitative and lacks objectivity. Intraoperative endoscopy would be a useful tool to assess the 

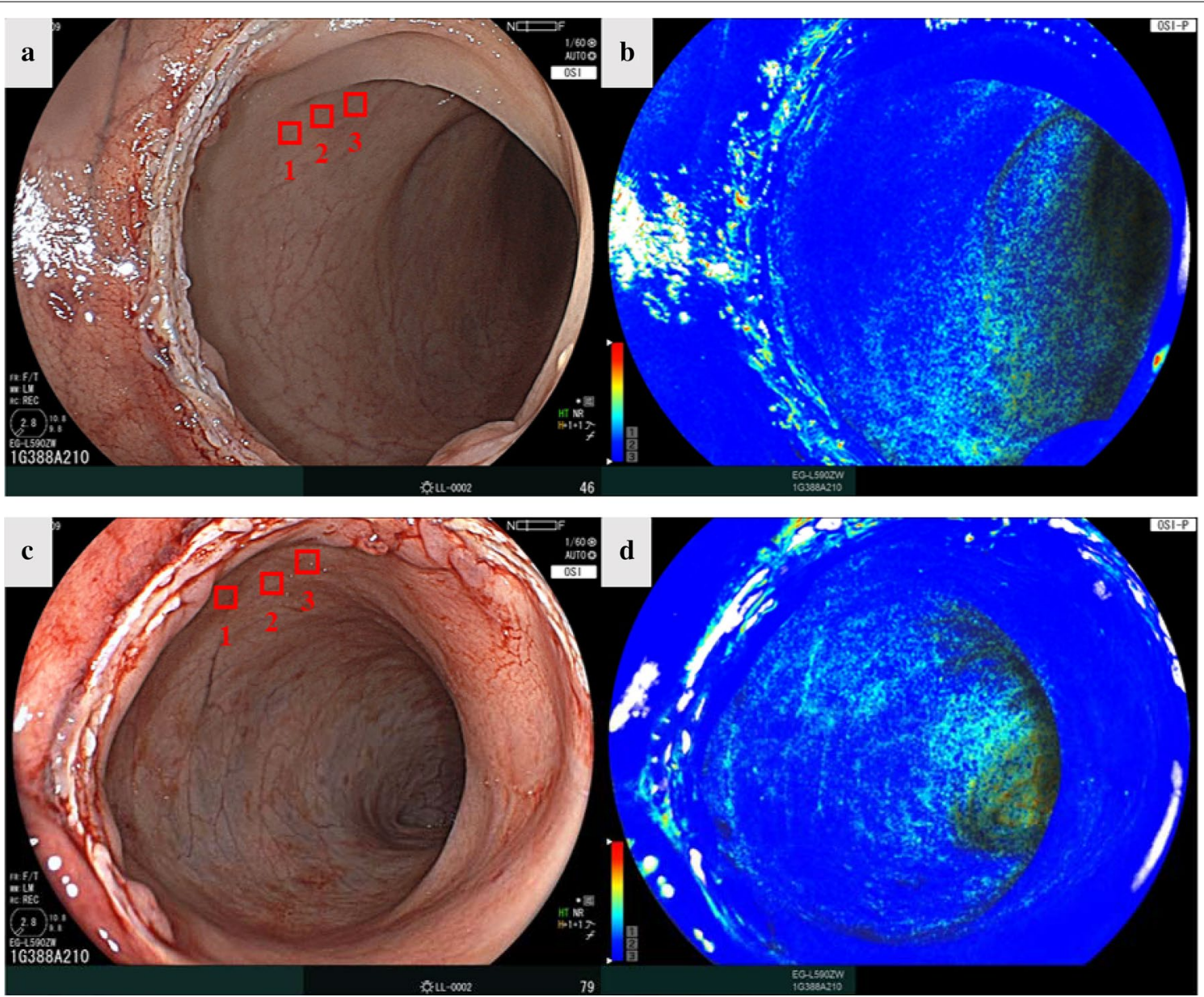

Fig. 5 a White light image one minute after $\mathrm{CRA}$ ligation. $\mathbf{b} \mathrm{StO}_{2}$ map 1 min after $\mathrm{CRA}$ ligation. c White light image 30 min after $\mathrm{CRA}_{\text {ligation. } \mathbf{d} \mathrm{StO}}$ map 30 min after CRA ligation. CRA cranial rectal artery, $\mathrm{StO}_{2}$ tissue oxygen saturation

macroscopic status of the anastomosis and air leakage status along with enabling simultaneous intraluminal visualization [6-9]. In addition, it also enables therapeutic intervention to control the intraluminal bleeding [6]. However, to the best of our knowledge, there is no available endoscope system that can assess these factors simultaneously.

The novel endoscope system used in this study has been developed to assess bowel perfusion by deriving $\mathrm{StO}_{2}$ maps, which is one of the measures of tissue perfusion $[11,12]$. Since this system does not require administration of the fluorescent substance, it can be performed anytime to assess bowel perfusion and can be performed repeatedly as well. In addition, a qualitative evaluation can be performed at a speed of $15 \mathrm{fps}$ almost in real time. However, $\mathrm{StO}_{2}$ level cannot be acquired in real time because it is necessary to extract the stored still image and analyze it using dedicated analysis software in order to perform quantitative evaluation. Therefore, some improvements in the system are required for practical application.

This new endoscope system can also be used for the postoperative assessment of the anastomosis. 


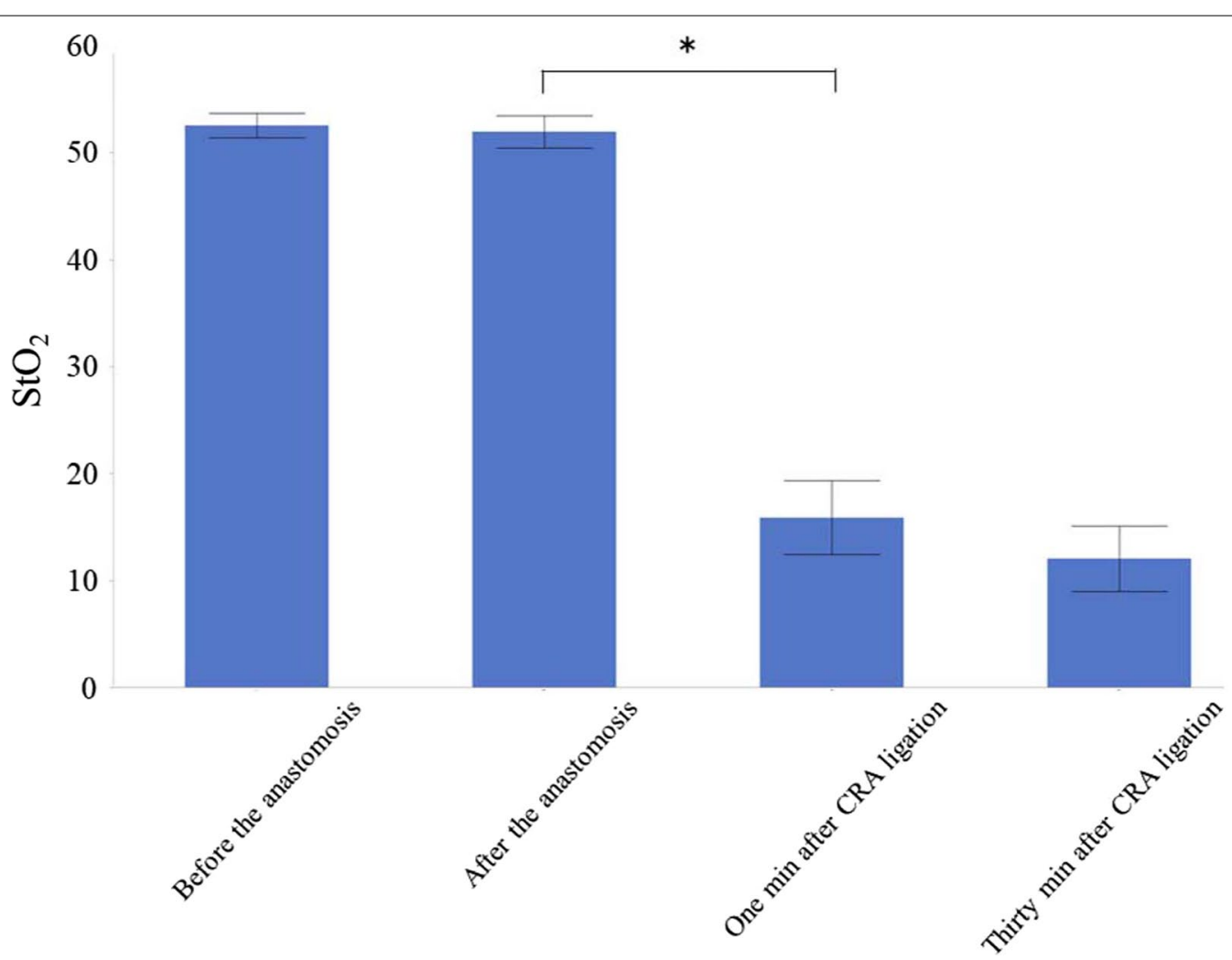

Fig. 6 The average $\mathrm{StO}_{2}$ levels compared among the different time points. *Indicates a $p$ value $<0.05$. $C R A$ cranial rectal artery, StO $\mathrm{O}_{2}$ tissue oxygen saturation

Postoperative endoscopic evaluation of the anastomosis could enable the rapid use of the appropriate treatment [14].

\section{Conclusions}

This novel OSI technology seems to be a promising method to assess not only the macroscopic status of, but also ischemic changes in the anastomosis immediately after ligation of the feeding vessel in an experimental model.

\section{Abbreviations}

CRA: Cranial rectal artery; Fps: Frames per second; ICG-FA: Indocyanine green fluorescence angiography; IP: Image processing; OSI: Oxygen saturation imaging; RGB: Red-green-blue; $\mathrm{SpO}_{2}$ : Saturation of percutaneous oxygen; $\mathrm{StO}_{2}$ : Tissue oxygen saturation; WLI: White light imaging.

\section{Acknowledgements}

Not applicable.

\section{Authors' contributions}

$\mathrm{HH}$ made substantial contributions to the conception and design, acquisition of data, and analysis and interpretation of data. $\mathrm{HH}$ drafted the manuscript, and NT and MI substantively revised it. All authors read and approved the final manuscript.

\section{Funding}

MI was supported by grants from the National Cancer Center Research and Development Fund (31-A-9). The funding source had no role in the design, practice or analysis of this study.

\section{Availability of data and materials}

The datasets used and/or analysed during the current study are available from the corresponding author on reasonable request.

\section{Ethics approval and consent to participate}

The study protocol was approved by the Committee for Ethics of Animal Experimentation of the National Cancer Center (Japan) (K18-022). Experiments were performed according to the Guidelines for Animal Experiments (Science Council of Japan: Guidelines for Proper Conduct of Animal Experiments, 2006) and Animal Research: Reporting of In Vivo Experiments (ARRIVE) guidelines.

\section{Consent for publication}

Not applicable. 


\section{Competing interests}

$\mathrm{HH}, \mathrm{NT}$ and Ml received experimental animals and borrowed an endoscope system from FUJIFILM Corporation, Kanagawa, Japan. There are no other competing interests to declare.

\section{Author details}

1 Department of Colorectal Surgery, National Cancer Center Hospital East, 6-5-1 Kashiwanoha, Kashiwa, Chiba 277-8577, Japan. ${ }^{2}$ Surgical Device Innovation Office, NEXT Medical Device Innovation Center, National Cancer Center Hospital East, Kashiwa, Japan.

Received: 14 July 2020 Accepted: 14 October 2020

Published online: 22 October 2020

\section{References}

1. Kang CY, Halabi WJ, Chaudhry OO, et al. Risk factors for anastomotic leakage after anterior resection for rectal cancer. JAMA Surg. 2013:148:65-71.

2. Snijders HS, Wouters MW, van Leersum NJ, et al. Meta-analysis of the risk for anastomotic leakage, the postoperative mortality caused by leakage in relation to the overall postoperative mortality. Eur J Surg Oncol. 2012:38:1013-9.

3. Mirnezami A, Mirnezami R, Chandrakumaran K, Sasapu K, Sagar P, Finan $P$. Increased local recurrence and reduced survival from colorectal cancer following anastomotic leak: systematic review and meta-analysis. Ann Surg. 2011;253:890-9.

4. Mongin C, Maggiori L, Agostini J, Ferron M, Panis Y. Does anastomotic leakage impair functional results and quality of life after laparoscopic sphincter-saving total mesorectal excision for rectal cancer? A casematched study. Int J Colorectal Dis. 2014;29:459-67.
5. Kingham TP, Pachter HL. Colonic anastomotic leak: risk factors, diagnosis, and treatment. J Am Coll Surg. 2009;208:269-78.

6. Nachiappan S, Askari A, Currie A, Kennedy RH, Faiz O. Intraoperative assessment of colorectal anastomotic integrity: a systematic review. Surg Endosc. 2014;28:2513-30.

7. Ishihara S, Watanabe T, Nagawa H. Intraoperative colonoscopy for stapled anastomosis in colorectal surgery. Surg Today. 2008;38:1063-5.

8. Yang SY, Han J, Han YD, et al. Intraoperative colonoscopy for the assessment and prevention of anastomotic leakage in low anterior resection for rectal cancer. Int J Colorectal Dis. 2017;32:709-14.

9. Shibuya N, Matsuda T, Yamashita K, et al. Clinical significance of intraoperative colonoscopy for anastomotic assessment in rectal cancer surgery. Anticancer Res. 2019;39:5761-5.

10. Shen R, Zhang Y, Wang T. Indocyanine green fluorescence angiography and the incidence of anastomotic leak after colorectal resection for colorectal cancer: a meta-analysis. Dis Colon Rectum. 2018;61:1228-34.

11. Kaneko K, Yamaguchi H, Saito T, et al. Hypoxia imaging endoscopy equipped with laser light source from preclinical live animal study to firstin-human subject research. PLoS ONE. 2014;10:e99055.

12. Saito T, Yamaguchi $\mathrm{H}$. Optical imaging of hemoglobin oxygen saturation using a small number of spectral images for endoscopic application. J Biomed Opt. 2015;20:126011.

13. Kilkenny C, Browne WJ, Cuthill IC, Emerson M, Altman DG. Improving bioscience research reporting: the ARRIVE guidelines for reporting animal research. PLoS Biol. 2010;8:e1000412.

14. Phitayakorn R, Delaney CP, Reynolds HL, et al. Standardized algorithms for management of anastomotic leaks and related abdominal and pelvic abscesses after colorectal surgery. World J Surg. 2008;32:1147-56.

\section{Publisher's Note}

Springer Nature remains neutral with regard to jurisdictional claims in published maps and institutional affiliations.
Ready to submit your research? Choose BMC and benefit from:

- fast, convenient online submission

- thorough peer review by experienced researchers in your field

- rapid publication on acceptance

- support for research data, including large and complex data types

- gold Open Access which fosters wider collaboration and increased citations

- maximum visibility for your research: over $100 \mathrm{M}$ website views per year

At BMC, research is always in progress.

Learn more biomedcentral.com/submissions 